\title{
Compact Multi-Port Power Combination/Distribution With Inherent Bandpass Filter Characteristics
}

\author{
Uwe Rosenberg, Senior Member, IEEE, Mehdi Salehi, Smain Amari, and Jens Bornemann, Fellow, IEEE
}

\begin{abstract}
Compact multi-port power combiners and dividers with filter characteristics are introduced. These components advantageously combine the functions of power distribution and filtering in a single component, thus reducing the number of components in a system and improving overall system performance. The design principle, which is entirely based on filter theory, is first presented for a symmetric filtering four-port combiner/divider and is then extended to an eight-port network. Measurements on a symmetric all-metal waveguide four-port prototype show excellent agreement with simulations and validate the general design approach. The experimental response of an asymmetric substrate integrated waveguide eight-port network agrees reasonably well with simulations and demonstrates that the basic four-port element can be extended to very compact multi-port combiner/dividers to cope with a variety of systems requirements.
\end{abstract}

Index Terms-Filter synthesis, multiports, power combiner, power divider, substrate integrated waveguide (SIW), waveguide filters.

\section{INTRODUCTION}

$\mathbf{M}$ ULTI-PORT combiner/distribution networks are widely used in modern communications systems. They are, for example, employed for the combination of several high-power signals (e.g., [1]), Butler matrices, as well as for beam-forming networks within an antenna subsystem (e.g., [2]). Thus, these multi-port networks are generally aiming at low dissipation losses to accommodate optimal systems properties and high power-handling capability. Therefore, they are often implemented in waveguide technology. A realization with substrate integrated waveguide (SIW) technology can provide an attractive compromise with reasonable dissipation loss on one hand and compact size and low production cost on the other.

Manuscript received April 25, 2014; revised July 31, 2014; accepted September 22, 2014. Date of publication October 15, 2014; date of current version November 03, 2014. This work was supported in part by the Natural Science and Engineering Research Council of Canada (NSERC) and under the TELUS Grant in Wireless Communications.

U. Rosenberg is with Mician Global Engineering GbR, 28195 Bremen, Germany (e-mail: uwe.rosenberg@ieee.org).

M. Salehi was with the Department of Electrical and Computer Engineering, University of Victoria, Victoria, BC, Canada V8W 2Y2. He is now with the Department of Electrical and Computer Engineering, University of Waterloo, Waterloo, ON, Canada N2L 3G1 (e-mail: msalehiv@uwaterloo.ca).

S. Amari is with the Department of Electrical and Computer Engineering, Royal Military College of Canada, Kingston, ON, Canada K7K 7B4 (e-mail: smain.amari@rmc.ca).

J. Bornemann is with the Department of Electrical and Computer Engineering, University of Victoria, Victoria, BC, Canada V8W 2Y2 (e-mail: j.bornemann@ieee.org).

Color versions of one or more of the figures in this paper are available online at http://ieeexplore.ieee.org.

Digital Object Identifier 10.1109/TMTT.2014.2361345
The principle implementation of both variants (metal waveguide and SIW) is very similar. This means that the overall design of multi-port combiner networks considers convenient interconnections of several single/individual power-combiner components. Components that provide decoupled ports are commonly preferred because the general characteristics are largely independent of the matching properties of other components connected to the individual ports of the multi-port network. ${ }^{1}$ Consequently, these applications employ couplers, hybrids, rat-race rings, and/or magic tees (e.g., [3]-[5]) instead of simple power splitters (e.g., [3, pp. 345-359]). In addition, filtering capabilities are generally mandatory in communications system equipment.

Recently, there have been first implementations providing the combinations of power splitting and filtering functions [6]-[8]. One principle is based on coupled resonators; i.e., first, the common port couples directly the resonators of two separate identical filters [6], or secondly, the common port couples a common filter section with several resonators where the last resonator of this common section directly couples two resonators of individual path sections [7]. Another approach is based on the combination of simple power splitters combined with filter sections [8]. It should be noted that all these principles consider specific matching properties only at the common port, whereas matching properties at the distributing ports, as well as "isolation" between them, are principally resulting from the assigned power magnitudes; i.e., a 3-dB power splitting entails 6-dB return loss and "isolation" at/between the output ports. ${ }^{2}$ The matching properties at the common port as well as the determined power distribution are obtained only when all ports are (perfectly) terminated; i.e., a remarkable mismatch at only one of the ports will affect the overall performance (i.e., matching and power distribution). In addition, all these solutions are considering only a single common port for power distribution/combination.

The inherent drawbacks of these principles are obviated by the use of coupler solutions; they are providing matching and isolation properties for all ports. In addition, they provide the possibility of several input and output ports; i.e., it is well known that a single coupler provides two decoupled input and two output ports, respectively, that can be utilized for more complex and special designs such as Butler matrices.

\footnotetext{
${ }^{1}$ Note that in case of a simple branching/bifurcation, the overall properties depend on the matching properties at all ports.

${ }^{2}$ Without consideration of dissipative losses
} 
In the past, the coupler functions for such designs and the filtering have been dedicated to different components (e.g., [2]), and therefore, their designs have been treated and specified separately. Only recently have power dividers with inherent filter characteristics been introduced for microstrip circuitries, e.g., [9]-[11], low-temperature co-fired ceramic (LTCC) technology [12], and for a dielectric loaded cavity configuration [13].

Another general approach based on waveguide technology has been proposed in [14]. It builds on a basic four-port element with four resonators where each of the ports is coupled to one resonator while each resonator is coupled to two adjacent ones. An initial validation of this principle has been introduced by a power-combiner design in SIW technology, which exhibits a rat-race characteristic with second-order filter functions for the semi-signal transmission paths [15]. This paper uses the same basic concept for the basic building block, but follows up with theory, design guidelines, extensions to multi-port power combiners/dividers, transmission zeros, and implementation.

The application of this novel concept allows the merging of power dividers and filters so that only a single component will be required that simultaneously provides power distribution/ combination and filter characteristics, thus resulting in reduced number of components. Hence, the numbers of components and internal system interfaces as well as overall equipment costs are reduced while overall performance properties are improved.

Therefore, the aim of this paper is to introduce the advantageous application of this novel approach [15] to multi-port power distribution/combination networks with inherent higher order filter characteristics. The complete subsystem is designed in one part without any interconnections (internal interfaces) of individual coupler components such as in current state-ofthe-art multi-port realizations with distributed individual components. This permits a very compact design with well-defined characteristics and no need for post-assembly tuning or other compensation measures.

The validation of this approach is provided by two different prototypes. First, a four-port waveguide power divider with second-order filter function is designed, analyzed, and tested. The consideration of one port with an orthogonal alignment to the $H$-plane waveguide structure yields a symmetrical structure design. The computed and measured results of this design demonstrate that excellent performance properties are achievable, especially when structural symmetry of the basic four-port elements can be considered.

Secondly, an eight-port power distribution/combination network is prototyped in SIW technology. Due to the entirely planar SIW structure, this implementation possesses a certain inherent structural asymmetry, but still achieves isolation between all ports. The signals fed to two of the ports are equally distributed to four others, and the respective signal paths exhibit fifth-order filter characteristics. Each of the remaining two ports provides signal paths with third-order filter functions to only two assigned ones of the four ports. Good agreement between computed and measured characteristics validate the overall design approach.

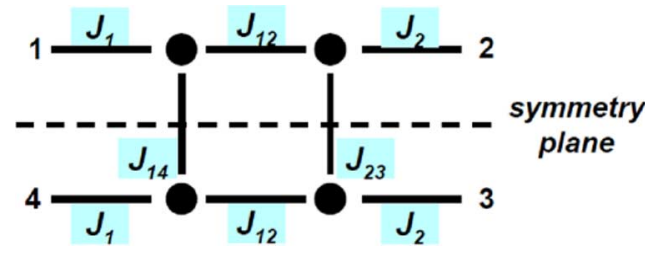

Fig. 1. Equivalent circuit of basic building block.

\section{THEORY AND BASIC FOUR-PORT DESIGN}

The basic building block of the class of components introduced in this paper is shown in Fig. 1. It consists of four resonators, shown as the black discs, coupled by admittance inverters $J_{i j}$. The structure is assumed symmetric with respect to the bisecting horizontal plane as shown. The analysis and design are carried out in the low-pass frequency domain where a resonator is represented by a unit shunt capacitor in parallel with a constant reactance $B_{i}$, which represents the frequency shift of the resonant frequency with respect to the center of the band (or a reference frequency). Naturally, this prototype is valid only for narrowband systems.

\section{A. Scattering Matrix}

The first step in the design is to relate the scattering matrix, or any other response function, of the structure to its adjustable parameters, which are taken as the frequency shifts and the inverters or coupling coefficients. To this end, we exploit the symmetry of the structure through its even and odd modes.

1) Even Mode: The even-mode results when ports 1 and 4 in Fig. 1 are excited by the same signal, say, $a_{1}$, and ports 2 and 3 by the same signal, say, $a_{2}$. The signals $a_{1}$ and $a_{2}$ are arbitrary. For this mode, the signals exiting ports 1 and 4 must be equal, and they are denoted by $b_{1}$. Similarly, the signals exiting ports 2 and 3 are equal and are denoted by $b_{2}$. The scattering matrix of this mode is defined by

$$
\left(\begin{array}{l}
b_{1} \\
b_{2}
\end{array}\right)=\left(\begin{array}{ll}
S_{11}^{e} & S_{12}^{e} \\
S_{21}^{e} & S_{22}^{e}
\end{array}\right)\left(\begin{array}{c}
a_{1} \\
a_{2}
\end{array}\right)
$$

The analysis can be further simplified by placing open circuits in the symmetry plane and keeping only half of the circuit. This sub-circuit then consists of inverter $J_{1}$ in cascade with resonator 1, from which half of inverter $J_{14}$ is dangling, in cascade with inverter $J_{12}$, resonator 2 , from which half of inverter $J_{23}$ is dangling, and finally, inverter $J_{2}$. An expedient way to analyze two-port networks of this type is through the $A B C D$ matrix. Following this procedure, we obtain

$$
\begin{aligned}
\left(\begin{array}{cc}
A_{e} & B_{e} \\
C_{e} & D_{e}
\end{array}\right)= & \left(\begin{array}{cc}
0 & \frac{j}{J_{1}} \\
j J_{1} & 0
\end{array}\right) \\
& \times\left(\begin{array}{cc}
1 & 0 \\
s+j B_{1}+j J_{14} & 1
\end{array}\right)\left(\begin{array}{cc}
0 & \frac{j}{J_{12}} \\
j J_{12} & 0
\end{array}\right) \\
& \times\left(\begin{array}{cc}
1 & 0 \\
s+j B_{3}+j J_{23} & 1
\end{array}\right)\left(\begin{array}{cc}
0 & \frac{j}{J_{2}} \\
j J_{2} & 0
\end{array}\right)
\end{aligned}
$$

Carrying out the multiplications, we get (3a)-(3d) shown at the bottom of the following page. 
The scattering matrix of this mode follows from the standard relations between the $A B C D$ matrix and the scattering matrix, normalized to unity in this case, as given in the Appendix.

2) Odd Mode: The odd mode can be analyzed similarly to the even mode. Short circuits are placed in the symmetry plane, keeping only half of the circuit. The $A B C D$ matrix of the remaining half of the circuit is obtained from the $A B C D$ matrix of the even mode by changing the signs of $J_{23}$ and $J_{14}$. The result is

$$
\begin{aligned}
\left(\begin{array}{cc}
A_{o} & B_{o} \\
C_{o} & D_{o}
\end{array}\right)= & \left(\begin{array}{cc}
0 & \frac{j}{J_{1}} \\
j J_{1} & 0
\end{array}\right) \\
& \times\left(\begin{array}{cc}
1 & 0 \\
s+j B_{1}-j J_{14} & 1
\end{array}\right)\left(\begin{array}{cc}
0 & \frac{j}{J_{12}} \\
j J_{12} & 0
\end{array}\right) \\
& \times\left(\begin{array}{cc}
1 & 0 \\
s+j B_{3}-j J_{23} & 1
\end{array}\right)\left(\begin{array}{cc}
0 & \frac{j}{J_{2}} \\
j J_{2} & 0
\end{array}\right) .
\end{aligned}
$$

By multiplying out these matrices, we get (5a)-(5d), shown at the bottom of this page. Once the $A B C D$ matrices of the even and odd modes are known, we construct the scattering matrices of each mode and then that of the overall structure according to the relationship

$$
S=\left(\begin{array}{llll}
\frac{S_{11}^{e}+S_{11}^{o}}{2} & \frac{S_{12}^{e}+S_{12}^{o}}{2} & \frac{S_{12}^{e}-S_{12}^{o}}{2} & \frac{S_{11}^{e}-S_{11}^{o}}{2} \\
\frac{S_{12}^{e}+S_{12}^{o}}{2} & \frac{S_{22}^{e}+S_{22}^{o}}{2} & \frac{S_{11}^{e}-S_{11}^{o}}{2} & \frac{S_{12}^{e}-S_{12}^{o}}{2} \\
\frac{S_{12}^{e}-S_{12}^{o}}{2} & \frac{S_{11}^{e}-S_{11}^{o}}{2} & \frac{S_{22}^{e}-S_{22}^{o}}{2} & \frac{S_{12}^{e}+S_{12}^{o}}{2} \\
\frac{S_{11}^{e}-S_{11}^{o}}{2} & \frac{S_{12}^{e}-S_{12}^{o}}{2} & \frac{S_{12}^{e}+S_{12}^{o}}{2} & \frac{S_{11}^{e}-S_{11}^{o}}{2}
\end{array}\right) .
$$

When the elements of this scattering matrix are calculated, it is found that their denominator is of order 4 in the complex frequency $s$ with leading term $s^{4}$. However, interesting cancellations take place when additional constraints are imposed, as we now discuss.

3) Case With $J_{14}=-J_{23}, J_{1}=J_{2}$, and $B_{1}=B_{3}$ : An examination of the equations relating the $A B C D$ parameters to scattering parameters in the Appendix shows that, under these conditions, the denominators of the scattering matrices of the even and odd modes are identical. This leads to a reduction in the order of the denominator of the scattering parameters of the overall structure from 4 to 2 , shown in (7)-(10) at the bottom of the following page.

The scattering parameters given by (10) show that port 1 is completely isolated from port 3 and so is port 2 from port 4 . This is a direct consequence of the symmetry of the structure and the phase reversal in $J_{14}$ and $J_{23}$. Equation (9) shows that the transmission between ports 1 and 4 and that between ports 2 and 3 are equal in magnitude, but always $180^{\circ}$ out of phase. The transmission coefficients between ports 1 and 2 and between ports 3 and 4 are equal both in magnitude and phase, as shown by (8). Finally, the relative coupling as given by $S_{21}$ and $S_{14}$ is determined by the ratio $J_{12} / J_{14}$.

\section{B. Design for $J_{14}=-J_{23}, J_{1}=J_{2}$, and $B_{1}=B_{3}$}

Since this building block consists of coupled resonators, it can be designed similarly to coupled-resonator filters. The symmetry is further exploited to reduce the number of dimensions that must be determined. It is, however, worth pointing out that the class of second-order responses that can be implemented is limited because of the reduced number of free parameters in the scattering parameters in (7)-(9). We consider a simple example to illustrate the procedure.

$$
\begin{aligned}
A_{e} & =-j \frac{J_{2}}{J_{1} J_{12}}\left(s+j B_{1}+j J_{14}\right) \\
B_{e} & =-j \frac{\left(s^{2}+j s\left(B_{1}+B_{3}+J_{23}+J_{14}\right)-\left(B_{1}+J_{14}\right)\left(B_{3}+J_{23}\right)\right)}{J_{1} J_{2} J_{12}}-j \frac{J_{12}}{J_{1} J_{2}} \\
C_{e} & =-j \frac{J_{1} J_{2}}{J_{12}} \\
D_{e} & =-j \frac{J_{1}}{J_{2} J_{12}}\left(s+j B_{3}+j J_{23}\right)
\end{aligned}
$$

$$
\begin{aligned}
A_{o} & =-j \frac{J_{2}}{J_{1} J_{12}}\left(s+j B_{1}-j J_{14}\right) \\
B_{o} & =-j \frac{\left(s^{2}+j s\left(B_{1}+B_{3}-J_{23}-J_{14}\right)-\left(B_{1}-J_{14}\right)\left(B_{3}-J_{23}\right)\right)}{J_{1} J_{2} J_{12}}-j \frac{J_{12}}{J_{1} J_{2}} \\
C_{o} & =C_{e}=-j \frac{J_{1} J_{2}}{J_{12}} \\
D_{o} & =-j \frac{J_{1}}{J_{2} J_{12}}\left(s+j B_{3}-j J_{23}\right)
\end{aligned}
$$


Let us assume that target second-order transmission coefficients of the following forms are sought:

$$
\begin{aligned}
S_{21} & =\frac{j a_{21}}{s^{2}+d_{1} s+d_{2}} \\
S_{41} & =\frac{j a_{41}}{s^{2}+d_{1} s+d_{2}} .
\end{aligned}
$$

The constants $a_{21}, a_{41}, d_{1}$, and $d_{2}$ are real with the denominator being a Hurwitz polynomial. The first step is to relate the parameters of the model, $J_{1}, J_{12}$, and $B_{1}$ to these constants be matching (8) and (11), as well as (9) and (12). The resulting constraints are

$$
\begin{aligned}
& 2 J_{1}^{2} J_{12}=a_{21} \\
& 2 J_{1}^{2} J_{14}=a_{41} \\
& 2\left(j B_{1}^{2}+J_{12}^{2}\right)=d_{1} \\
& 2 j J_{12}^{2} B_{1}-B_{1}^{2}+J_{14}^{2}+J_{12}^{2}+J_{1}^{4}=d_{2} .
\end{aligned}
$$

For an arbitrary set $d_{1}, d_{2}, a_{21}$, and $a_{41}$, there may be no solution to (13a)-(13d) with real values of $J_{1}, J_{12}, J_{14}$, and $B_{1}$. This is best illustrated by using an example of a second-order 3-dB Chebyshev response.

The transmission coefficients are given by

$$
S_{21}=S_{41}=\frac{j 3.5169}{s^{2}+2.9996 s+4.9987} .
$$

This expression is obtained from the standard pole-zero decomposition of a second-order Chebyshev filter with a ripple constant $\varepsilon=0.1005$ and a return loss of $20 \mathrm{~dB}$.

From (13c), we see that $B_{1}=0$. From (13a) and (13b), and the fact that $a_{21}=a_{41}$, we see that $J_{12}=J_{14}\left(J_{23}=-J_{14}\right)$. Under this condition, (13c) and (13d) give $J_{12}=J_{14}=1.2247$ and $J_{1}=1.1891$. However, these values do not satisfy (13a) and (13b) since $2 J_{1}^{2} J_{12}=3.4633$, which is slightly different from $a_{21}=3.5169$. It is also straightforward to confirm that the roots of $S_{11}$ as given by (7) are located at $s= \pm 1$ instead of \pm 0.7071 for a second-order two-pole Chebyshev response. This example confirms that there is no exact solution for a pure Chebyshev response for this configuration.

Fortunately, very good approximate solutions can be found. For the same example, an optimized solution leads to $B_{1}=$

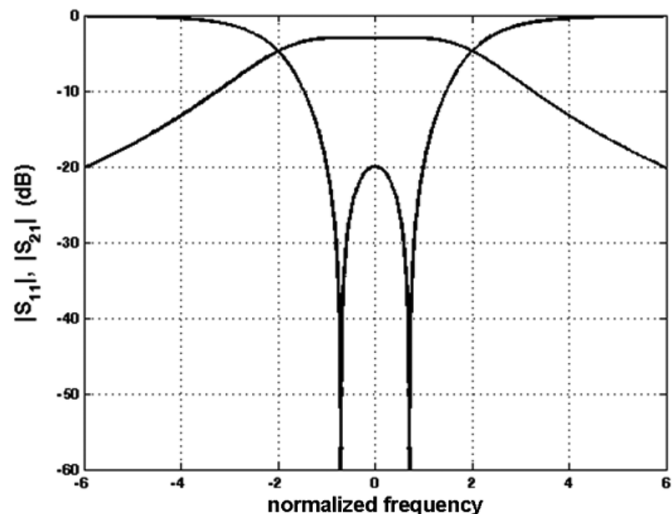

(a)

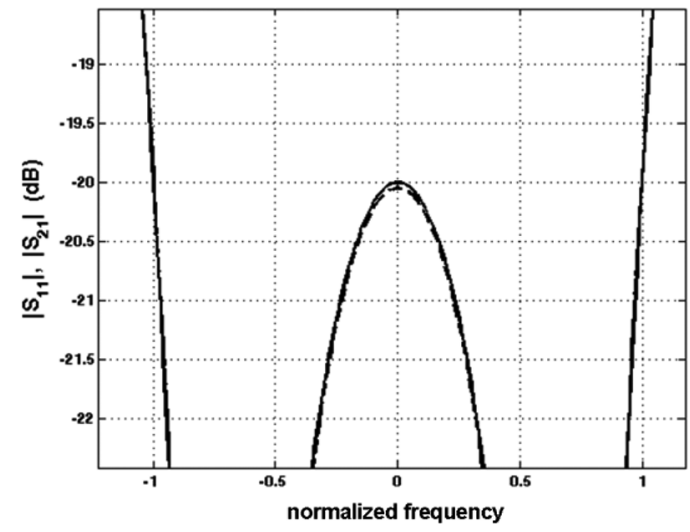

(b)

Fig. 2. Responses of the central building block with $B_{1}=0, J_{12}=J_{14}=$ $1.1717, J_{1}=1.2246$ (solid lines) and the second-order Chebyshev "filter" in (14) (dashed lines). (a) Wideband response. (b) Expanded view of the passband.

$0, J_{12}=J_{14}=1.1717$, and $J_{1}=1.2246$. A plot of $S_{11}$ and $S_{21}$ for these values along with the transmission coefficient given by (14) is given in Fig. 2(a). The agreement between the two responses is very good over the entire frequency range. In fact, the difference between the two responses is not visible in this figure. An expanded view of the passband does show the difference [see Fig. 2(b)].

Once the values of the coupling coefficients (inverters) are known, the design of the central building block follows standard techniques of coupled-resonator filters. Since these are well documented, e.g., [16], they are not repeated here.

and

$$
\begin{aligned}
& S_{11}=S_{22}=S_{33}=S_{44}=\frac{s^{2}+2 j s B_{1}-B_{1}^{2}+J_{14}^{2}+J_{12}^{2}-J_{1}^{4}}{s^{2}+2 s\left(j B_{1}+J_{12}^{2}\right)+2 j J_{12}^{2} B_{1}-B_{1}^{2}+J_{14}^{2}+J_{12}^{2}+J_{1}^{4}} \\
& S_{12}=S_{21}=S_{34}=S_{43}=\frac{2 j J_{1}^{2} J_{12}}{s^{2}+2 s\left(j B_{1}+J_{12}^{2}\right)+2 j J_{12}^{2} B_{1}-B_{1}^{2}+J_{14}^{2}+J_{12}^{2}+J_{1}^{4}} \\
& S_{14}=S_{41}=-S_{23}=-S_{32}=\frac{2 j J_{1}^{2} J_{14}}{s^{2}+2 s\left(j B_{1}+J_{12}^{2}\right)+2 j J_{12}^{2} B_{1}-B_{1}^{2}+J_{14}^{2}+J_{12}^{2}+J_{1}^{4}}
\end{aligned}
$$

$$
S_{13}=S_{31}=S_{24}=S_{42}=0
$$




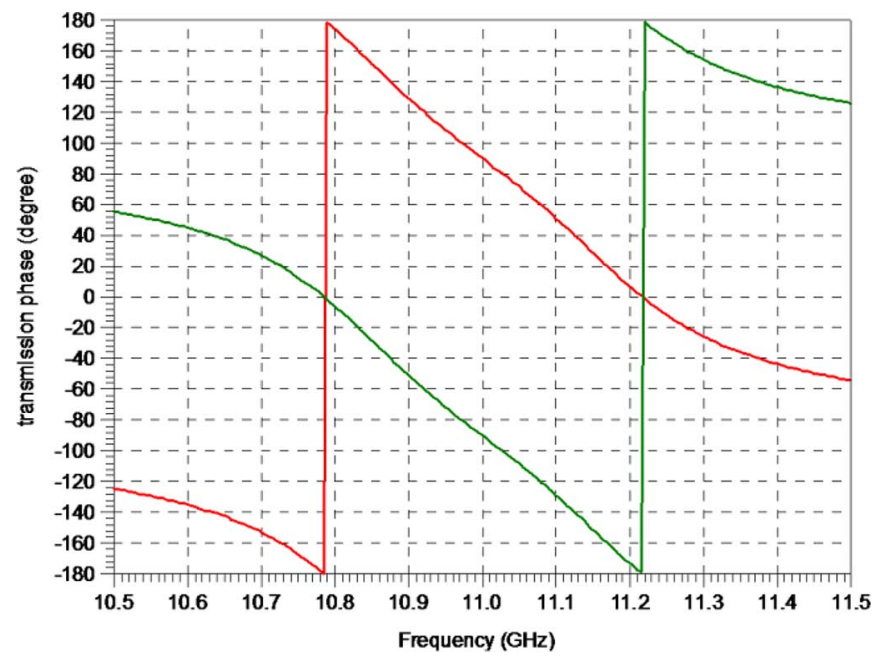

Fig. 3. Phase characteristics of a basic four-port design (cf. Fig. 1) at $11 \mathrm{GHz}$ (150-MHz bandwidth, return loss $26 \mathrm{~dB}$ ); red (in online version): $\varangle S_{21}=$ $\varangle S_{41}=\varangle S_{43}$; green (in online version): $\varangle S_{23}$.

\section{Design CONSIDERATIONS FOR MultiPORT NETWORKS}

It is obvious that the general concept introduced in this paper is not only restricted to the designs of four-ports as presented for the basic building block. The almost arbitrary combination of basic four-port elements with additional resonator/filter sections allows the design of very compact multi-port subsystems that can accommodate a variety of system requirements.

In multiport subsystems applications, aspects regarding the implementation of special filter characteristics and phase relations may become important for the individual signal paths. Consequently, the realization of a frequency-selective power distribution subsystem has to consider the convenient design and combination of the basic building blocks, possibly with additional filter sections. Hence, the inherent characteristics of basic structures are decisively important.

\section{A. Phase Characteristics}

As introduced above, the basic building block exhibits four ports. It provides the splitting of a signal fed to one of the ports into semi portions occurring at two dedicated other ports, while the fourth port is isolated. All ports are matched within the passband and the power distribution will not be impaired in principle by the matching properties terminating the ports. Thus, the principle characteristic within the passband is comparable to that of a magic tee or rat-race ring, e.g., [17]. It depends on whether for the input signal at the feeding port, the power splitting is in-phase $\left(0^{\circ}\right)$ or out-of-phase $\left(180^{\circ}\right)$. Considering the basic structure of Fig. 1 with $J_{12}=J_{14}=-J_{23}$, an in-phase power splitting is achieved for an input signal at port $1\left(S_{21}=S_{41} ; S_{31}=0\right)$ and port $4\left(S_{41}=S_{43} ; S_{42}=0\right)$. Out-of-phase power splitting occurs for signals fed to port 2 $\left(S_{21}=-S_{32} ; S_{42}=0\right)$ and port $3\left(S_{43}=-S_{32} ; S_{31}=0\right)$, respectively. The analyzed phase characteristics for this basic building block design at $11 \mathrm{GHz}$ with $150-\mathrm{MHz}$ bandwidth are shown in Fig. 3.

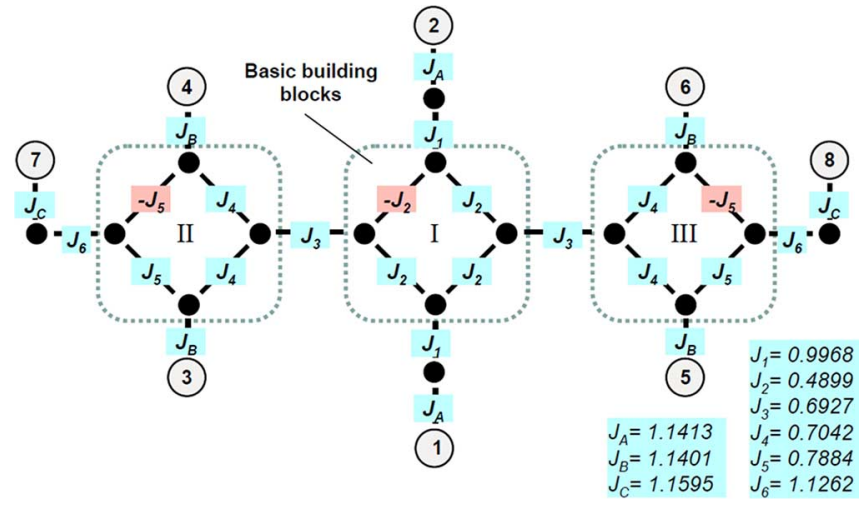

Fig. 4. Schematic of eight-port power divider/combiner; gray disks with numbers indicate ports; basic-block numbering (from left to right) II, I, III.

Consequently, the design of a multiport power distribution network - consisting of the direct combination of several basic building blocks - has to carefully consider the phasing relations of the individual blocks. In other words, for an overall design, the configuration of the individually combined blocks is decisively important to satisfy specific phasing requirements as necessary, for example, for beam-forming networks. However, the relative phasing relation of the individual paths does not only depend on the inherent phase relation of the basic blocks. The transmission phase of each path also depends on the number of resonators and the transformation properties of the resonators within individual paths. For example, an extra $\mathrm{TE}_{101}$ mode resonator within a path transforms the phase by $180^{\circ}$.

\section{B. Phase Design Example: Eight-Port Power Divider ${ }^{3}$}

The example of an eight-port frequency-selective power divider (cf. schematic in Fig. 4) demonstrates the general design approach. The subsystem consists of 16 coupled resonators, 12 of which are forming three basic building blocks (II, I, III) that are directly interconnected with couplings $J_{3}$. One additional resonator is attached each to ports 1 and 2 . Two other resonators are considered at ports 7 and 8 .

This structure has been optimized to satisfy operation at $11 \mathrm{GHz}$ (bandwidth of $400 \mathrm{MHz}$ ) with the following characteristics (cf. coupling values in Fig. 4).

- 6-dB power distribution of input signals at port 1 (and 2) to output ports 3-6. These signal paths exhibit five resonators yielding a fifth-order Chebyshev filter response [cf. Fig. 5(a)]. Considering the paths with the input signal at port 1, all output signals are in-phase. A signal fed to port 2 exhibits in-phase signals at ports 5 and 6 , while the output signals at port 3 and 4 are out-of-phase (due to the negative coupling $\left(-J_{2}\right)$ in block I), as shown in Fig. 4). Thus, $S_{31}=S_{41}=S_{51}=S_{61}$ and $\left(-S_{32}=-S_{42}=S_{52}=\right.$ $\left.S_{62}\right)$.

- $3-\mathrm{dB}$ power-splitting functions of input signals at port 7 (and 8 ) to output ports 3 and 4 (5 and 6). The respective signal paths have three resonators, thus providing third-

\footnotetext{
${ }^{3}$ The description considers the divider case for simplicity- of course the design can be also operated in the reverse direction as a combiner.
} 


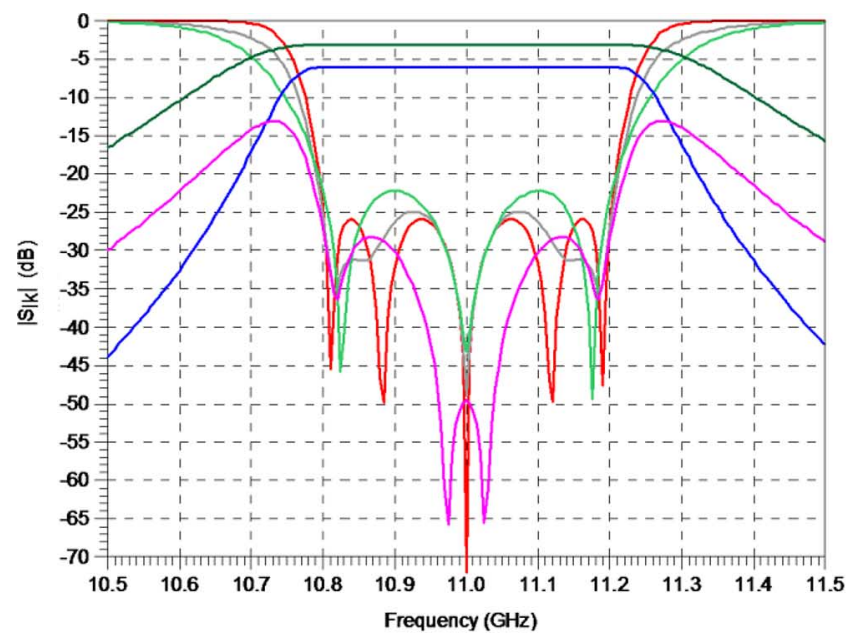

(a)

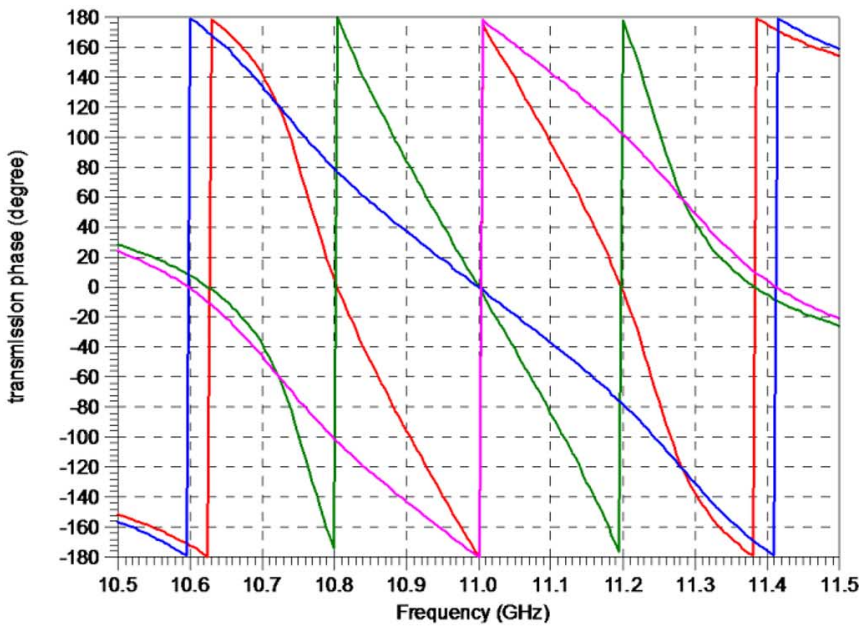

(b)

Fig. 5. Computed characteristics of the eight-port network shown in Fig. 4. (a) Magnitude, red (in online version): $S_{11}=S_{22}$; green (in online version): $S_{33}=S_{44}=S_{55}=S_{66}$; turquoise (in online version): $S_{77}=S_{88} ;$ blue (in online version): $S_{31}=S_{41}=S_{51}=S_{61}=S_{32}=S_{42}=S_{52}=S_{62}$; grey (in online version): $S_{73}=S_{74}=S_{85}=S_{86}$; magenta (in online version): $S_{43}=S_{65}$. (b) Transmission phases, red (in online version): $\varangle S_{31}=\varangle S_{41}=$ $\varangle S_{51}=\varangle S_{61}=\varangle S_{52}=\varangle S_{62}$; green (in online version): $\varangle S_{32}=\varangle S_{42}$; blue (in online version): $\varangle S_{74}=\varangle S_{86}$; magenta (in online version): $\varangle S_{73}=$ $\varangle S_{85}$.

order Chebyshev responses [cf. Fig. 5(a)]. Due to the negative coupling $\left(-J_{5}\right)$ in block II (III), the output signal at port $3(5)$ is in-phase and out-of-phase at port 4 (6) [cf. Fig. 5(b)]. Thus, $S_{73}=-S_{74}$ and $S_{85}=-S_{86}$.

- All ports are matched within the passband:

- Return losses at ports 1 and 2 are according to the fifthorder filter function.

- Return losses at ports 7 and 8 are according to the thirdorder filter function.

- Return losses at ports 3-6 result from the combination of the third- and fifth-order responses because these ports are connected to both paths.

Thus, $S_{11}, S_{22}, S_{33}, S_{44}, S_{55}, S_{66}, S_{77}$, and $S_{88}<$ $-23 \mathrm{~dB}$

- The isolation of the different ports allows simultaneously the independent operation of the different power-splitter

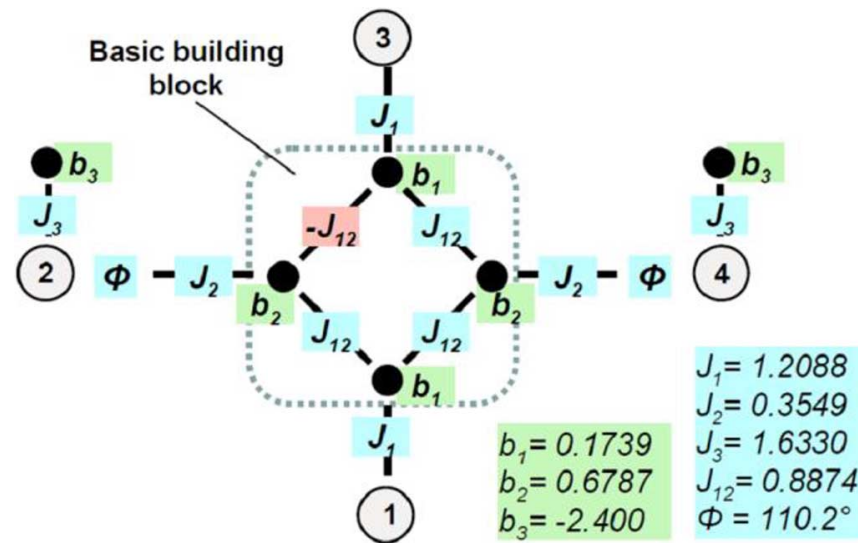

Fig. 6. Basis building block with extracted pole resonators (coupling scheme with normalized parameters).

functions. In addition, imperfect matching properties of interface equipment at the ports will only slightly impair the respective filter and power divider functions. Perfect isolation is (theoretically) achieved for $S_{21}, S_{71}, S_{81}, S_{72}$, and $S_{82}$. Perfect isolation properties cannot be achieved for $S_{43}$ and $S_{65}$ since ports 3 and 4 , as well as 5 and 6 are sharing two different filter functions. However, as Fig. 5(a) shows, reasonable isolation is achieved for these ports $\left(S_{43}, S_{65}<-25 \mathrm{~dB}\right)$, which is commonly sufficient for most applications. Note that perfect isolation would be possible by considering two extra resonators between port 7 (8) and block II (III) for the realization of the same filter functions as for the fifth-order paths of the equipment (e.g., between ports 1 and 3 ).

It is obvious that the third- and fifth-order filter characteristics for the different paths with the same equiripple passband exhibit different $3-\mathrm{dB}$ bandwidths and selectivity responses (cf., e.g., Fig. 5(a), $S_{73}$ and $S_{31}$, respectively). For the above design, both responses exhibit a $400-\mathrm{MHz}$ equiripple passband, the third-order response with $22 \mathrm{~dB}$ and the fifth-order characteristic with 26-dB return loss, respectively [see Fig. 5(a)]. It should be noted that the responses are according to their theoretical filter characteristics, which includes $3-\mathrm{dB}$ bandwidths and selectivities. ${ }^{4}$

This example demonstrates that in case of complex power distribution networks, careful considerations are necessary for the determination of the couplings to obtain the respective output signals. Note that the negative coupling of the basic building block can be assigned arbitrarily to one of the four couplings. This provides a certain freedom for the overall design to accommodate specific phase requirements for different paths.

\section{Filter Functions With Transmission Zeros}

The aim of high spectrum efficiency commonly yields the need of high-selectivity requirements close to the passband. Standard filter designs accommodate these demands with tailored characteristics considering transmission zeros.

\footnotetext{
${ }^{4}$ Identical filter functions for all paths are possible with two extra resonators considered at each of the ports 7 and 8 .
} 


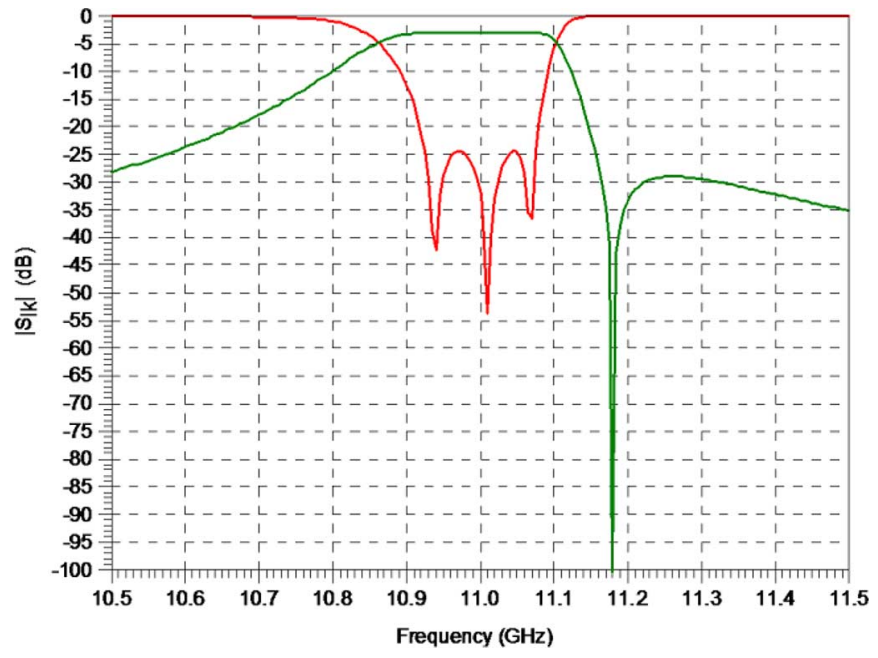

Fig. 7. Computed responses of configuration in Fig. 6 (with extracted pole); red (in online version): $S_{11}, S_{22}, S_{33}, S_{44}$; green (in online version): $S_{21}, S_{41}, S_{32}, S_{43}$; perfect isolation: $S_{31}=S_{42}=0$.

The basic building block introduced in Section II exhibits a second-order Chebyshev filter response between all transmission paths. As shown by the example above, the filter order of a multiport network is increased due to the direct combination of several blocks, as well as using extra resonators directly coupled to the ports of the basic blocks. However, the sequential coupling of resonators always yields Chebyshev responses.

This general approach, however, is based on coupled resonators in the same way as applied for standard filter designs. Thus, extra filter sections that are used for the implementation of transmission zeros (singlets, doublets, etc., as used in modular filter designs [16], [18], [19]) can be employed within the basic blocks or within the overall multiport network.

Two examples are introduced to demonstrate the variety of possibilities. The schematic of the first example is depicted in Fig. 6. In this case, a basic building block is extended symmetrically at two of its ports with extracted pole resonators. The parameters (couplings, resonances, and phases between the extracted pole cavities and the assigned resonators of the block) have been determined to satisfy third-order filter characteristics at $11 \mathrm{GHz}$ (bandwidth $150 \mathrm{MHz}$, return loss $25 \mathrm{~dB}$ ) with a transmission zero at $11.18 \mathrm{GHz}$ (design considerations similar to [20]). The computed characteristics of this configuration (depicted in Fig. 7) validate this general approach.

In the second example, the basic building block is symmetrically associated with two doublets, i.e., each doublet is directly coupled to a dedicated resonator of the block (cf. schematic in Fig. 8). Thus, fourth-order filter responses with one transmission zero are obtained for the transmission paths. The couplings and resonances are determined to satisfy a filter characteristic at $11 \mathrm{GHz}$ (bandwidth $200 \mathrm{MHz}$, return loss $25 \mathrm{~dB}$ ) with a transmission zero at $11.2 \mathrm{GHz}$. The computed results of this design in Fig. 9 also verify this design possibility.

Thus, it should be noted that the introduced multiport powerdivider approach can be extended by suitable filter sections for the implementation of advanced filter characteristics with transmission zeros. These sections will be directly associated with the basic building blocks coupled between different blocks or

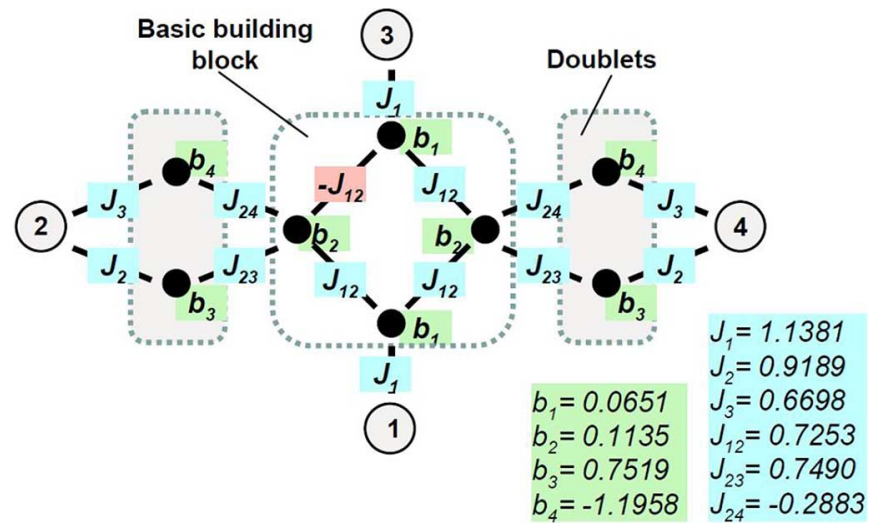

Fig. 8. Basic building block extended with doublets; coupling scheme with normalized parameters.

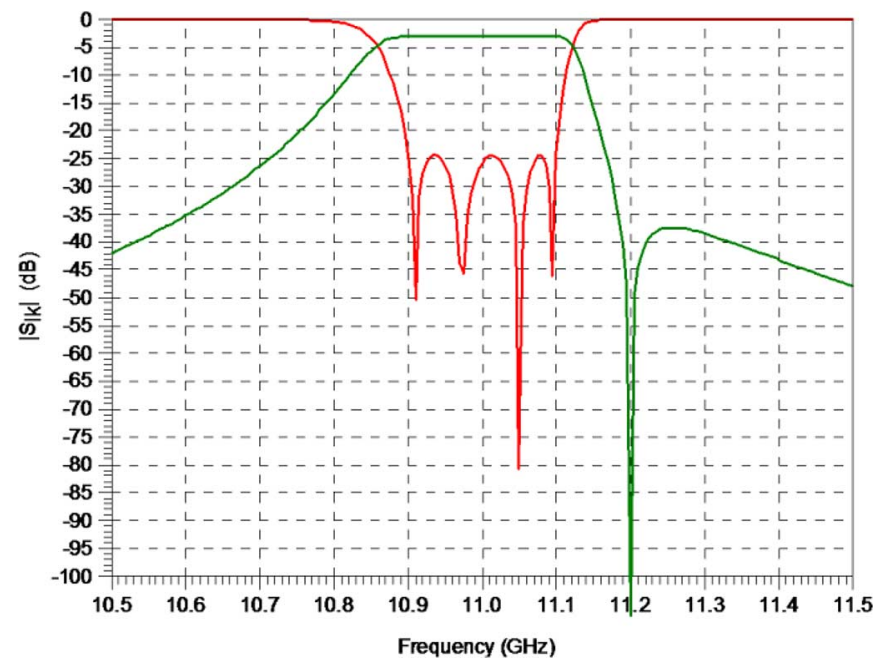

Fig. 9. Computed responses of basic building block with two doublets according to Fig. 8; red (in online version): $S_{11}=S_{22}=S_{33}=S_{44}$; green (in online version): $S_{21}=S_{41}=S_{32}=S_{43}$; perfect isolation: $S_{31}=S_{42}=0$.

between blocks and interface ports. They have to be considered in the overall design since they are improving the filter functions of the dedicated paths. The examination also includes their effects on the path transmission phases in applications where the phase properties are of special interest. The implementation of extracted pole solutions is only useful close to interface ports since they require specific phase relations that are commonly realized by transmission line sections.

\section{General Remarks Regarding Implementation}

The above characteristics of the equivalent circuits are based on the exact values of the dedicated identical parameters (considering also the exact out-of phase conditions of the related couplings, i.e., the symmetry of the structure determined in Section II-B). However, there are commonly special constraints for the implementation of physical microwave structures. One is related to resonator arrangements allowing the realization of the required couplings between dedicated resonators. Another aspect applies to the realization of positive and negative couplings, especially with identical value, but opposite sign. These constraints entail usually different structures and dimensions, respectively, for the designs of the individual elements. In 


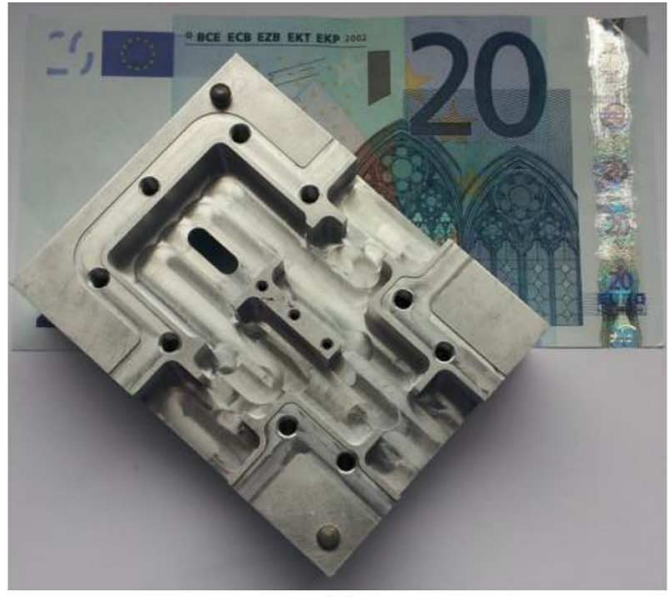

(a)

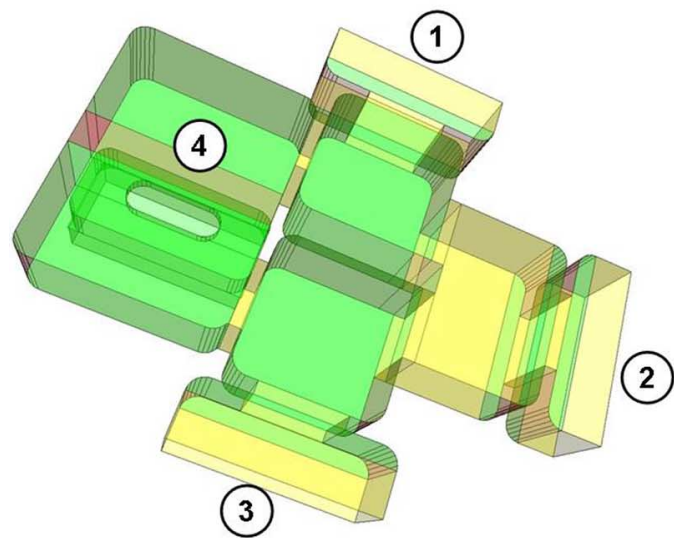

(b)

Fig. 10. Photograph of the opened four-port power combination/distribution component in: (a) waveguide technology and (b) port designation.

other words, the design of the structure of each individual element (resonator, iris) accommodates the realization of the determined parameter of the equivalent circuit to satisfy the required symmetry "electrically." However, the approximation of the exact values is only achieved in the vicinity of the center frequency, which is also known from the equivalent circuit representation in standard filter designs. Especially in case of different realizations of the same element value, there will be small differences in the variation of amplitude and phase characteristics versus frequency.

In the present approach, the effect of these small differences is especially observed by the impairment of the perfect isolation between the assigned ports.

Considering for example the design of the basic four-port structure introduced in Section IV (Figs. 10 and 11). This implementation exhibits single-plane symmetry across ports 1 and 3 . The negative coupling is accomplished by a symmetrically coupled $\mathrm{TE}_{201}$ cavity, yielding identical dimensions of the positive and negative coupling iris. The intercavity irises coupling resonator 1 with resonators 2 and 4 are also identical. Due to this symmetry, the respective transmission signal paths from port 1 to port 2 and 4 (as well as from port 3 to 2 and 4 ) exhibit identical responses. Thus, almost perfect isolation $(>50 \mathrm{~dB})$ between ports 1 and 3 is observed. (Note that the "non-ideal" value

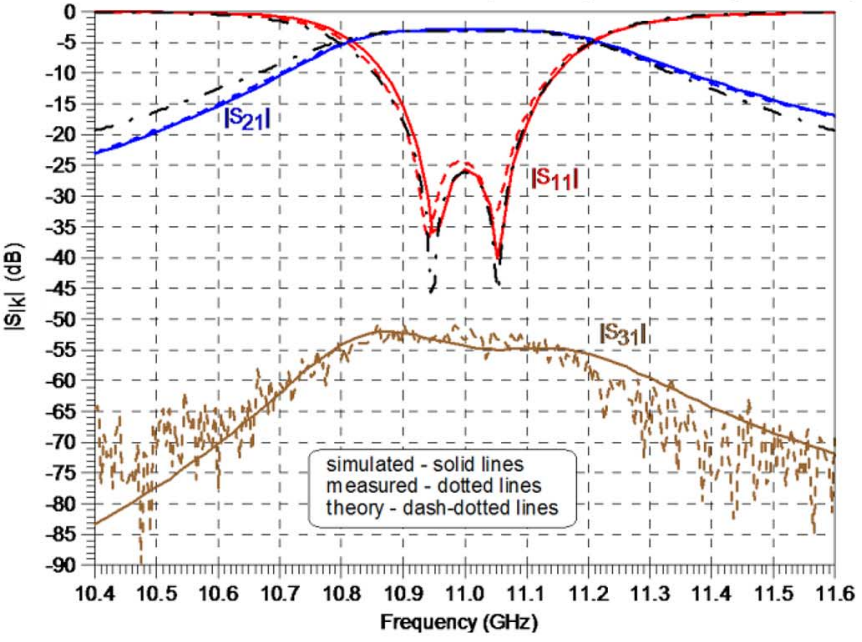

(a)

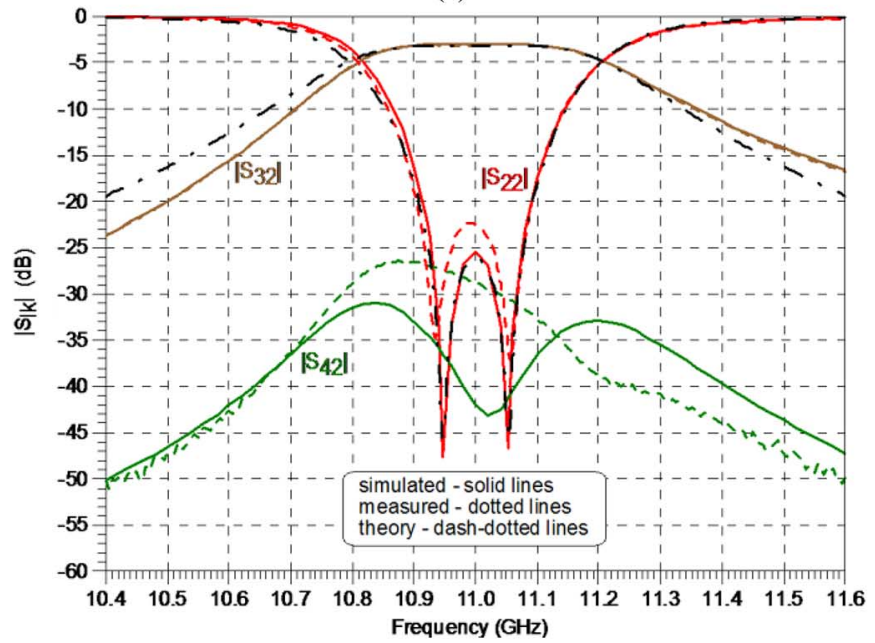

(b)

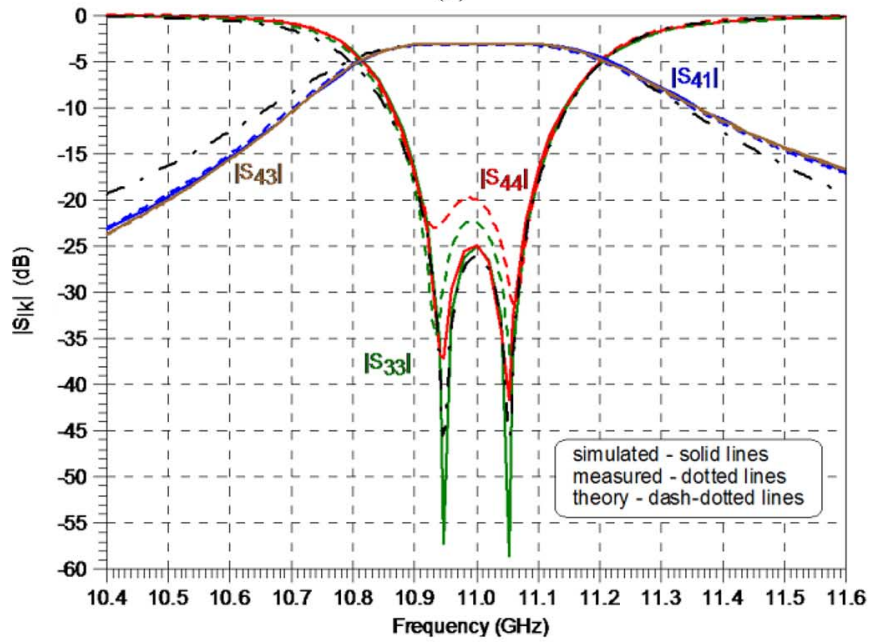

(c)

Fig. 11. Comparisons between theoretical, simulated, and measured scattering parameters for the four-port prototype in waveguide technology.

of $>50 \mathrm{~dB}$ of the electromagnetic (EM) simulation can be attributed to small imperfections of the modeling.)

Compared to this symmetrical port situation of the structure, a noticeable impairment of the isolation between ports 2 and 4 is observed (cf. responses of simulation in Fig. 11(b), $S_{42}>35 \mathrm{~dB}$ 


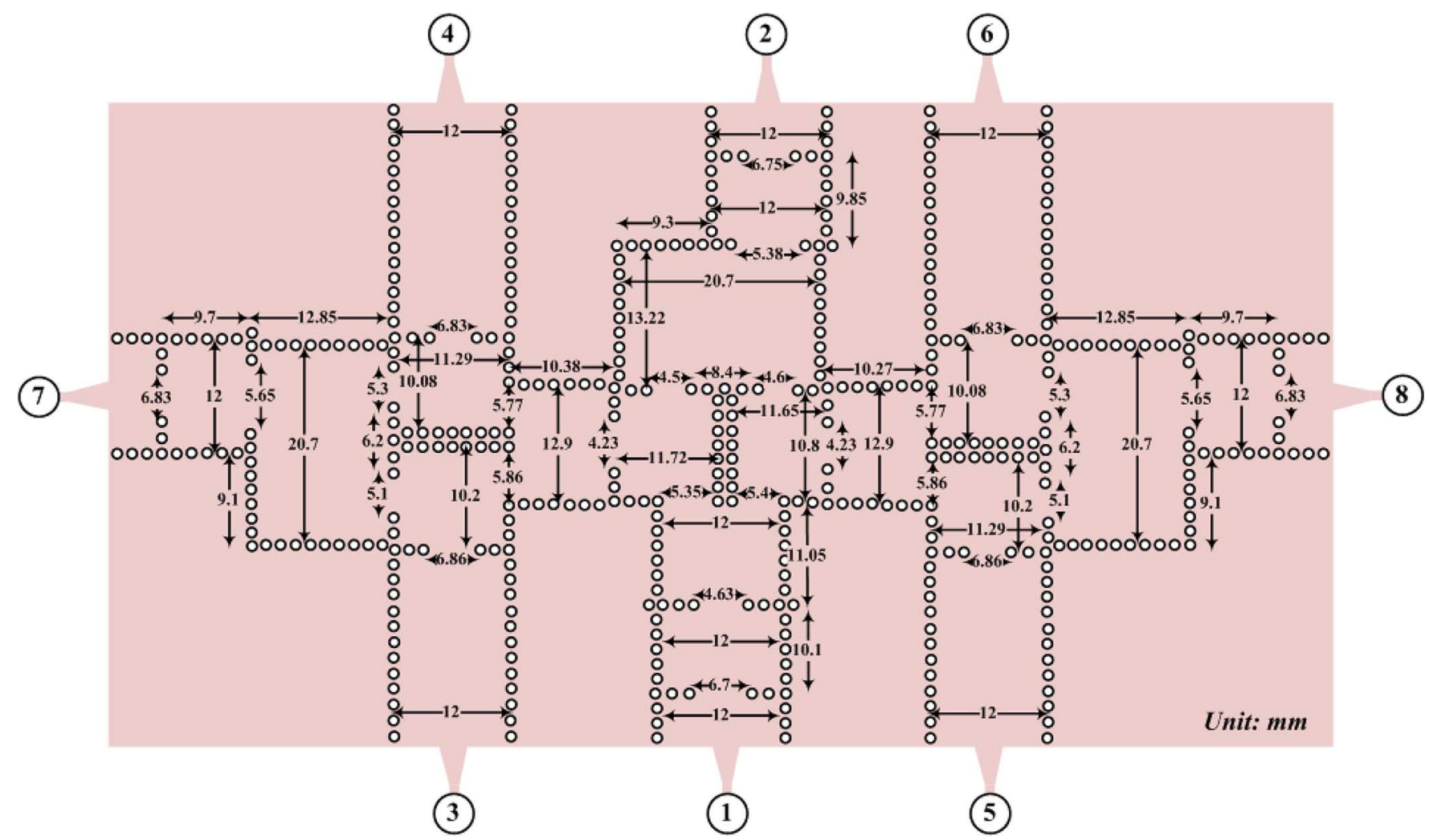

Fig. 12. Eight-port combiner/divider network in SIW technology with dimensions in millimeters [actual number of via-holes according to the inset in Fig. 15(a)].

within the passband). There is no symmetry for these ports, i.e., the different paths of the semi-signals through the $\mathrm{TE}_{101}$ and $\mathrm{TE}_{201}$ cavities (towards ports 1 and 3 , respectively) cause small differences in the transfer characteristics. The comparison of the computed amplitude and phase characteristics of the associated paths $\left(S_{21}=S_{41}\right.$ and $\left.S_{32}=S_{43}\right)$ within the passband $\left(f_{0} \pm\right.$ $75 \mathrm{MHz}$ ) exhibits a maximum amplitude imbalance of $0.02 \mathrm{~dB}$ and a maximum phase imbalance of $0.7^{\circ}$. These deviations are mainly the reason for the nonperfect isolation of the assigned ports.

As this examination demonstrates, implementations with symmetrical configurations provide the advantage of almost perfect characteristics of the dedicated paths, i.e., no amplitude and phase imbalance and perfect isolation. However, this is not a general prerequisite for this approach, i.e., realizations without any structural symmetry are feasible. Although such designs will exhibit small deviations of the amplitude and phase characteristics for the individual path responses within the passband, they still provide high-performance properties as the eight-port design example below proves.

\section{RESULTS}

This section presents the implementation of the design theory and considerations for two different multi-port power combination/distribution components.

The first circuit is a single-plane symmetric four-port waveguide circuit that satisfies the conditions outlined in Section II-B. It is designed for a bandwidth of $150 \mathrm{MHz}$, centered at $11 \mathrm{GHz}$ with a return loss of $26 \mathrm{~dB}$. The normalized parameters for this design are $B_{1}=0, J_{12}=J_{14}=-J_{23}=$
1.4874 , and $J_{1}=1.4072$. Fine optimization is performed in the $\mu$ Wave Wizard. A photograph of the opened prototype is shown in Fig. 10(a). The model of the complete configuration in $\mu$ Wave Wizard including port designation is depicted in Fig. 10(b). The component is realized as a structural part that is completed with a flat lid-made from aluminum using CNC milling techniques. Note that of port 4 in Fig. 10(b), only the aperture is seen in Fig. 10(a), where this port points into the page.

The basic design consists of an $H$-plane configuration of four cavities. Each cavity exhibits couplings to one port and the two adjacent cavities according to the equivalent circuit in Fig. 1. Three of the cavities are utilizing the $\mathrm{TE}_{101}$ mode resonances while one cavity (the one associated with port 4) is operated with the $\mathrm{TE}_{201}$ resonance mode. This allows the utilization of the coupling transformation properties (similar as in [21]) of this mode to accommodate the prerequisite of $J_{23}=-J_{14}$ in the planar $H$-plane configuration. The three $\mathrm{TE}_{101}$ mode cavities exhibit iris couplings to the dedicated waveguide ports-all of which are realized within the $H$-plane waveguide configuration [cf. Fig. 10(a)]. The $\mathrm{TE}_{201}$ mode can be also coupled by an $H$-plane iris and waveguide port to maintain an overall $H$-plane configuration (as later considered for the eight-port SIW application), but this would yield a complete asymmetric structure - thus in such a case, the principle design demands of equal coupling values and resonances (cf. Section II-B) are satisfied electrically with individual dimensions of the elements, i.e., irises and cavities. However, the optimal "electrical" symmetry is principally only achieved in the close vicinity of the passband center frequency, yielding especially a deviation of the 
isolation performance caused by small phase and amplitude imbalances that are increasing from the center frequency toward the band edges.

For the present waveguide implementation, the inherent asymmetry of an $H$-plane coupled $\mathrm{TE}_{201}$ cavity is avoided by considering an interface from the top wall (port 4). This is an iris coupling associated with the interface waveguide and is located in the center of the top wall of the $\mathrm{TE}_{201}$ cavity-i.e., orthogonal to the $H$-plane iris couplings to the adjacent cavities [see Fig. 10(b)]. It is noticed that this implementation exhibits a single symmetry plane across ports 1 and 3 , while the plane across ports 2 and 4 is still asymmetric, which is finally also observed in the results.

Fig. 11 shows comparisons between the simulated and measured scattering parameters with port numbering depicted in Fig. 10(b). It is observed that the measurements agree very well with theoretical data obtained from the design procedure in Section II, as well as with simulations-except for the theoretically perfect isolation $\left[S_{31}\right.$ in Fig. 11(a) and $S_{42}$ in Fig. 11(b)] that could be hardly achieved in real implementations. (Note that the deviations of the selectivity of synthesis and simulated/measured results are quite similar to those known from typical $H$-plane filter designs.) The measured return loss at all four ports is better than $20 \mathrm{~dB}$, and the $3-\mathrm{dB}$ power combination/division capability is demonstrated. The maximum insertion loss down from the 3-dB level at the center frequency of $11 \mathrm{GHz}$ is only $0.16 \mathrm{~dB}$ for $S_{21}$ [see Fig. 11(a)] and $0.11 \mathrm{~dB}$ for $S_{32}$ [see Fig. 11(b)] as well as $S_{41}$ and $S_{43}$ [see Fig. 11(c)]. The measured isolation between ports 1 and 3 is better than $50 \mathrm{~dB}$ [see Fig. 11(a)] as a result of the symmetry plane across the respective ports. The measured isolation between opposite ports 2 and 4 is larger than $26 \mathrm{~dB}$, which is still an attractive figure for power-combiner applications. As mentioned above, this value is attributed to the asymmetry of the configuration across these ports. Nevertheless, the excellent results in measurements and simulations fully verify the design theory.

It is obvious that the general concept introduced in this paper is not only restricted to the designs of four-ports as presented here. The almost arbitrary combination of basic four-port elements with additional resonator/filter sections allows the design of very compact multi-port subsystems that can accommodate a variety of system requirements, as for example, those already introduced by the eight-port example in Section III. In order to demonstrate this point, the eight-port design above was used for a prototype implementation. However, instead of using metal waveguides, a SIW circuit was used. Fig. 12 shows the circuit layout and its dimensions.

Since a SIW is a planar technology, interfacing of the $\mathrm{TE}_{201}$-mode cavity in the waveguide version [see Fig. 10(b)] must now be placed in the same plane as all other ports. That makes the circuit completely asymmetric as observed in Fig. 12, and therefore, a larger impact on the isolation properties is expected. Although the symmetric design guidelines presented in Sections II and III can still be applied, they must accommodate an "electrical" symmetry considering individual designs of all elements, i.e., coupling irises and cavities, by an overall fine optimization. To accommodate the planar structure, the $\mathrm{TE}_{201}$-mode cavity of the basic design above is now coupled

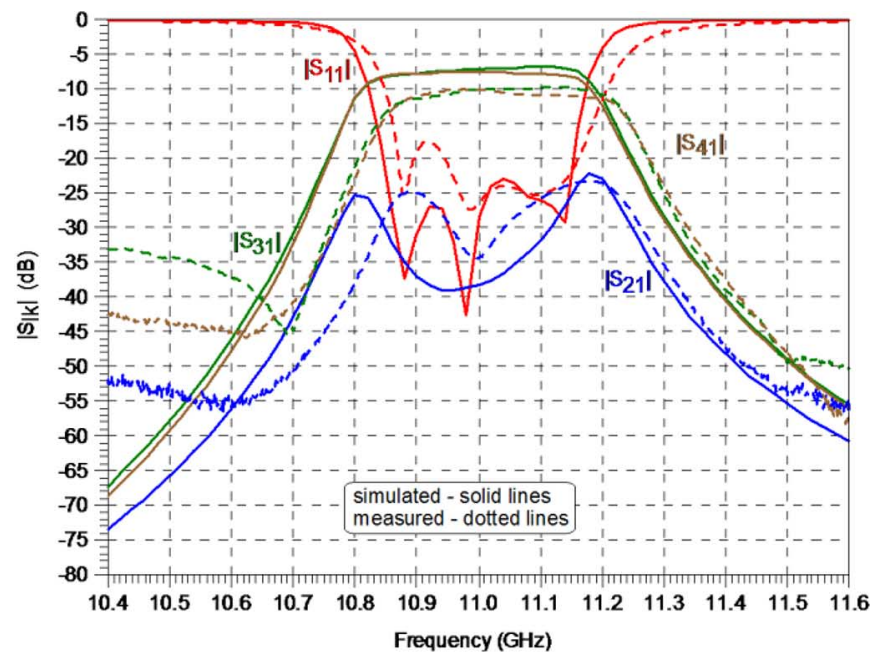

(a)

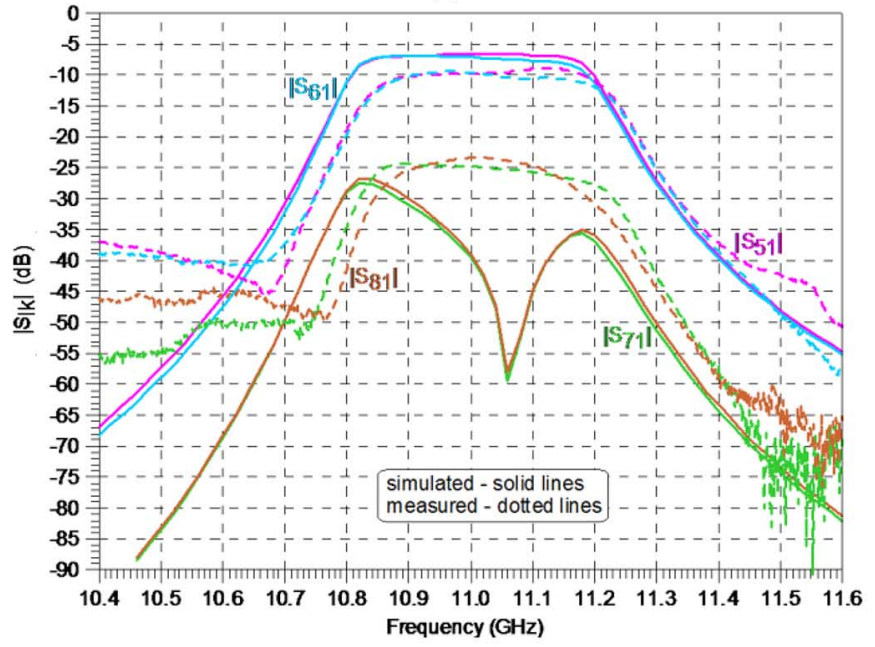

(b)

Fig. 13. Comparisons between simulated and measured scattering parameters for the eight-port prototype in SIW technology. (a) $\left|S_{11}\right|$ to $\left|S_{41}\right|$. (b) $\left|S_{51}\right|$ to $\left|S_{81}\right|$.

at the sidewall opposite to the iris couplings with the adjacent cavities of the basic four resonator block. An additional TE $\mathrm{T}_{101}$ cavity is considered between this coupling and the respective interface at all these ports - as can be seen in Fig. 12 at ports 2,7 , and 8 . Another extra resonator is also considered at port 1. It should be noted that these extra resonators do not principally have an influence on the coupling function, but they are increasing the filter functions of the dedicated paths (cf. Section III). The implementation depicted in Fig. 12 with alignment of the basic blocks to each other and the extra resonators corresponds to the equivalent circuit design in Fig. 4. Thus, the paths from port 1 (2) to 3-6 exhibit a fifth-order filter response with 6-dB power distribution while the paths from port 7 (8) to $3,4(5,6)$ have a third-order filter characteristic with $3-\mathrm{dB}$ power distribution.

Following the synthesis procedure, an all-dielectric waveguide component using commercial software such as $\mu$ Wave Wizard is designed for a bandwidth of $400 \mathrm{MHz}$ at center frequency of $11 \mathrm{GHz}$. The structure is then transferred to SIW technology according to equivalent waveguide widths reported in 


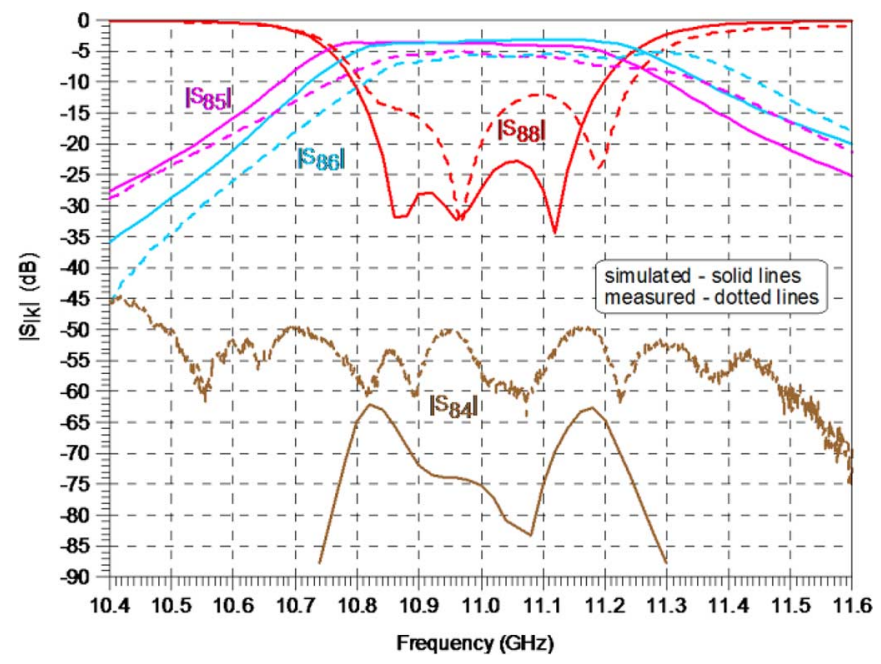

Fig. 14. Comparisons between simulated and measured scattering parameters for the eight-port prototype in SIW technology; $\left|S_{84}\right|$ to $\left|S_{88}\right|$.

[22]. However, fine optimization of all resonator and coupling parameters within HFSS is required to adjust for the transfer to SIW technology. In order to ease the many different requirements of this circuit, the optimization applied in this case favored amplitude over phase response.

The eight-port power divider/combiner is designed for an RT/Duroid 6002 substrate with $\varepsilon_{r}=2.94$, loss tangent 0.0012 , substrate thickness $h=0.508 \mathrm{~mm}$, conductor thickness $t=$ $35 \mu \mathrm{m}$, and conductivity $\sigma=5.8 \times 10^{7} \mathrm{~S} / \mathrm{m}$. The diameter of all via-holes is $1 \mathrm{~mm}$ with center-to-center spacing of $1.4 \mathrm{~mm}$ at all ports. For access with measurement equipment, all ports are interfaced with SIW-to-microstrip transitions according to [23].

In order to limit the number of figures, we present a selection of scattering parameters of the eight-port combiner/divider. Fig. 13 presents a comparison between simulation and measurements for parameters $\left|S_{k, 1}\right|$ with $1 \leq k \leq 8$. Reasonable agreement between simulations and measurements is obtained despite the fact that the original assumption of symmetry is violated. Measured return loss values in the order of $20 \mathrm{~dB}$ are demonstrated [see Fig. 13(a)], and the input power at port one is divided between ports $3-6$ while ports 2,7 , and 8 are isolated to at least $20 \mathrm{~dB}$.

Several discrepancies between measurements and simulations are observed. First of all, there is an added insertion loss of up to $3 \mathrm{~dB}$ compared to the simulations that include conductor and dielectric losses. This is comparable with many published SIW filters whose insertion losses are in the order of $2 \mathrm{~dB}$, e.g., [24]. Secondly, the measured return loss is worse than simulated and the isolation peak in $\left|S_{71}\right|$ and $\left|S_{81}\right|$ [see Fig. 13(b)] is not reproduced. And thirdly, a slight frequency shift towards higher frequencies is observed.

The frequency shift can in part be attributed to the tolerance of the dielectric constant, but mostly to the fact that a slightly larger drill size was used in the manufacturing process. That makes the via-holes larger and the resonators effectively smaller, thus shifting the frequency upwards. This may also be a reason for the degraded return loss as couplings are changed. The other deviations are mainly due to the measurement set

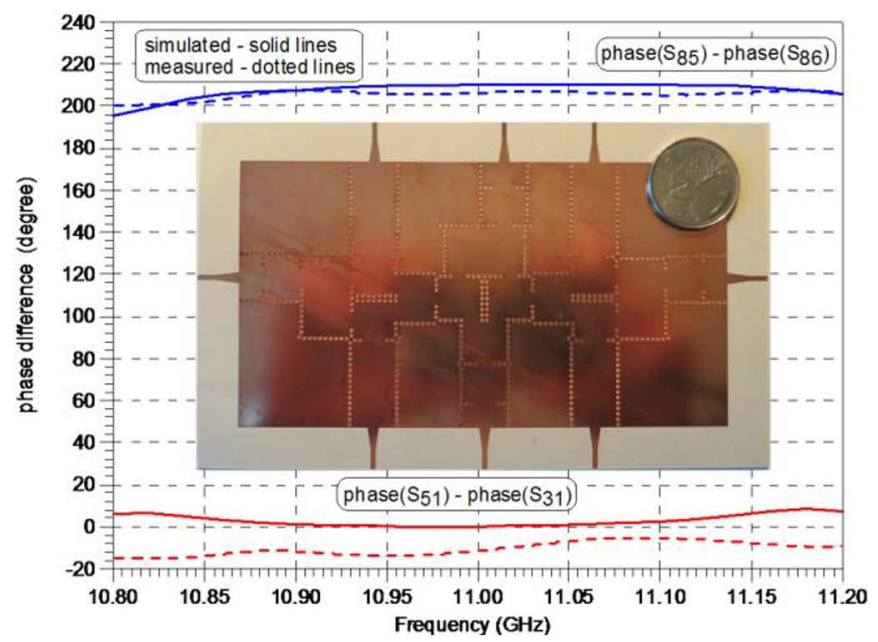

(a)

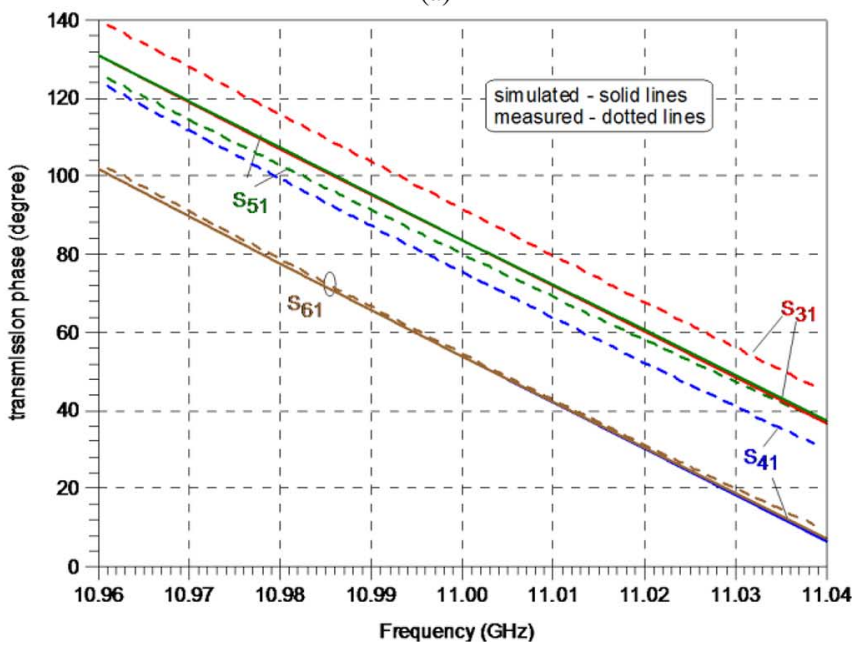

Fig. 15. (a) Photograph of the eight-port SIW prototype and comparisons between simulated and measured phase differences for ports with in-phase and out-of-phase power combination/division and (b) comparison between simulated and measured transmission phases.

up. Due to the size of the circuit (Fig. 12), a test fixture could not be used so that all measurements had to be performed with coaxial connectors attached to all ports and to all calibration standards. (TRL calibration standards were used to deembed the coax-to-microstrip-to-SIW transitions.) The different soldering of all such connectors influences the calibration, and thus the measurements. Moreover, only two ports can be deembedded at the same time while the remaining six ports are terminated with coaxial terminations. Thus, the reflections and losses caused by microstrip transitions and coaxial connectors fully influence the measurements, resulting in added reflections and estimated added losses of up to $1 \mathrm{~dB}$ within the circuit.

Fig. 14 shows the scattering parameters related to one of the side ports, port 8 . A signal fed into port 8 is divided between ports 5 and 6 , while all other ports such as port 4 [or port 1 from Fig. 13(b)] are isolated. Due to the lower filter order (three instead of five), the added loss is less than $1.5 \mathrm{~dB}$ compared to those parameters displayed in Fig. 13 (e.g., 3.8 dB simulated and $5.2 \mathrm{~dB}$ measured). The measurement of $\left|S_{84}\right|$ also shows the limitations in the dynamic range. 
The inset of Fig. 15(a) shows the SIW prototype. In order to explore the phase relationships within the passband, Fig. 15(a) displays comparisons between simulated and measured phase differences for in-phase and out-of-phase combining ports. Port 1 is divided in-phase between ports 3 and 5 (and ports 4 and 6). The agreement between measurements and simulations is reasonable with a maximum deviation of about $20^{\circ}$. A signal fed into port 8 is divided to ports 5 and 6 . This division is out-ofphase due to the $\mathrm{TE}_{201}$ mode in one of the cavities attached to port 8 . The agreement between measurements and simulations is very good, albeit the fact that the difference is $200^{\circ}$ instead of $180^{\circ}$.

In order to investigate these discrepancies, Fig. 15(b) displays the four transmission phases when feeding port 1 in the vicinity of the center frequency. It is observed that the simulated phases of $S_{31}$ and $S_{41}$ as well as those of $S_{51}$ and $S_{61}$ agree, but that there is about a $25^{\circ}$ difference between the two. This is a result of having optimization focus mainly on amplitude response. However, the phase measurements of $S_{31}$ and $S_{41}$ (to the left of the input port) are off by about $10^{\circ}$ and $20^{\circ}$, respectively, whereas those of $S_{51}$ and $S_{61}$ are in good agreement. This points to tolerances in the fabrication of the prototype on top of the soldering of connectors to the calibration standards. However, the overall performance is also a result of optimizing a completely asymmetric structure that cannot make use of all of the symmetry conditions assumed in Sections II and III. An all-metal waveguide version of this eight-port combiner/divider with the symmetrically coupled $\mathrm{TE}_{201}$ cavity would have ports 2,7 , and 8 (Fig. 12) pointing upwards [cf. port 4 in Fig. 10(b)], thus yielding improved performance properties due to the utilization of the one-plane structure symmetry of the basic building blocks.

\section{CONCLUSION}

The compact and frequency-selective multi-port power combiner/divider networks introduced in this paper present attractive solutions for modern communication systems. The approach is based on a basic four-port building block consisting of four directly coupled resonators. Multiport networks are realized by the direct coupling of several such building blocks. Moreover, additional cavities or complete filter sections (e.g., doublets, triplets, etc.) can be directly coupled between the building blocks or at their interfaces to accommodate special filter characteristics/requirements between individual ports. Consequently, this general concept offers a high degree of freedom in the design of multiport power divider/combiner networks with isolated/decoupled ports - providing additionally almost arbitrary filter functions between the ports. The design approach is entirely based on filter theory since all cavities of the structure are directly coupled. Thus, the solution does not only provide the merging of filter and combiner functions, it also facilitates very compact implementations, which is another important advantage. The measurements on the symmetric all-metal waveguide four-port prototype verify the design theory by excellent agreement with simulations. An eight-port prototype demonstrates the possible extension of the four-port element and the use of SIW technology. Due to limitations in port orientation in planar circuits, the unavoidable total asymmetry encountered in SIW yields a reduced performance compared with the all-metal waveguide circuit. However, the operational principle applies in approximation and provides a reasonable alternative when very compact planar circuitry is envisaged.

\section{APPENDIX}

The scattering parameters of the even/odd modes are given in terms of the respective normalized $A B C D$ parameters as

$$
\begin{aligned}
S_{11}^{e, o} & =\frac{A^{e, o}+B^{e, o}-C^{e, o}-D^{e, o}}{A^{e, o}+B^{e, o}+C^{e, o}+D^{e, o}} \\
S_{12}^{e, o} & =\frac{2\left(A^{e, o} D^{e, o}-B^{e, o} C^{e, o}\right)}{A^{e, o}+B^{e, o}+C^{e, o}+D^{e, o}} \\
S_{21}^{e, o} & =\frac{2}{A^{e, o}+B^{e, o}+C^{e, o}+D^{e, o}} \\
S_{22}^{e, o} & =\frac{-A^{e, o}+B^{e, o}-C^{e, o}+D^{e, o}}{A^{e, o}+B^{e, o}+C^{e, o}+D^{e, o}} .
\end{aligned}
$$

By using the expressions of the elements of the $A B C D$ matrix of the even mode as given by (3a)-(3d), the denominator of the scattering parameters of this mode becomes

$$
\begin{aligned}
A^{e}+ & B^{e}+C^{e}+D^{e} \\
= & a_{e} s^{2}+b_{e} s+c_{e} \\
a_{e}= & \frac{-j}{J_{1} J_{2} J_{12}} \\
b_{e}= & -j\left[\frac{J_{1}}{J_{2} J_{12}}+\frac{J_{2}}{J_{1} J_{12}}+\frac{j\left(B_{1}+B_{3}+J_{14}+J_{23}\right)}{J_{1} J_{2} J_{12}}\right] \\
c_{e}= & -j \frac{J_{12}}{J_{1} J_{2}}+\frac{J_{2}}{J_{1} J_{12}}\left(B_{1}+J_{14}\right) \\
& +j \frac{\left(B_{1}+J_{14}\right)\left(B_{3}+J_{23}\right)}{J_{1} J_{2} J_{12}}-j \frac{J_{1} J_{2}}{J_{12}} \\
& +\frac{J_{1}}{J_{2} J_{12}}\left(B_{3}+J_{23}\right) .
\end{aligned}
$$

Similarly, the denominator of the scattering parameters of the odd mode becomes

$$
\begin{aligned}
A^{o}+ & B^{o}+C^{o}+D^{o} \\
= & a_{o} s^{2}+b_{o} s+c_{o} \\
a_{o}= & \frac{-j}{J_{1} J_{2} J_{12}} \\
b_{o}= & -j\left[\frac{J_{1}}{J_{2} J_{12}}+\frac{J_{2}}{J_{1} J_{12}}+\frac{j\left(B_{1}+B_{3}-J_{14}-J_{23}\right)}{J_{1} J_{2} J_{12}}\right] \\
c_{o}= & -j \frac{J_{12}}{J_{1} J_{2}}+\frac{J_{2}}{J_{1} J_{12}}\left(B_{1}-J_{14}\right)+j \frac{\left(B_{1}-J_{14}\right)\left(B_{3}-J_{23}\right)}{J_{1} J_{2} J_{12}} \\
& -j \frac{J_{1} J_{2}}{J_{12}}+\frac{J_{1}}{J_{2} J_{12}}\left(B_{3}-J_{23}\right) .
\end{aligned}
$$

If we now assume that $B_{1}=B_{3}, J_{1}=J_{2}$ and $J_{14}=-J_{23}$, (A.2) gives

$$
\begin{aligned}
& a_{e}=\frac{-j}{J_{1}^{2} J_{12}} \\
& b_{e}=-j\left[\frac{2}{J_{12}}+\frac{2 j B_{1}}{J_{1}^{2} J_{12}}\right] \\
& c_{e}=-j \frac{J_{12}}{J_{1}^{2}}+\frac{2 B_{1}}{J_{12}}+j \frac{\left(B_{1}^{2}-J_{14}^{2}\right)}{J_{1}^{2} J_{12}}-j \frac{J_{1}^{2}}{J_{12}} .
\end{aligned}
$$


Under the same conditions, (A.3) becomes

$$
\begin{aligned}
& a_{o}=\frac{-j}{J_{1}^{2} J_{12}} \\
& b_{o}=-j\left[\frac{2}{J_{12}}+\frac{2 j B_{1}}{J_{1}^{2} J_{12}}\right] \\
& c_{o}=-j \frac{J_{12}}{J_{1}^{2}}+\frac{2 B_{1}}{J_{12}}+j \frac{\left(B_{1}^{2}-J_{14}^{2}\right)}{J_{1}^{2} J_{12}}-j \frac{J_{1}^{2}}{J_{12}} .
\end{aligned}
$$

Under these conditions, (A.4) and (A.5) show that $a_{e}=a_{o}$, $b_{e}=b_{o}$, and $c_{e}=c_{o}$. Since the denominators of the scattering parameters of the even and odd modes are identical, the overall scattering parameters have the same denominator as in (6). Each parameter has a denominator of second order. In the general case, each denominator of the scattering parameters of the central block is of order 4 .

\section{ACKNOWLEDGMENT}

The authors would like to thank Prof. J. Hesselbarth, University of Stuttgart, Stuttgart, Germany, for taking care of the precise manufacturing of the four-port power combiner, and Prof. M. Schneider and H. Masemann, both with the University of Bremen, Bermen, Germany, for accurate measurement of the waveguide component.

\section{REFERENCES}

[1] R. N. Simons, E. G. Wintucky, J. D. Wilson, and D. A. Force, "Ultra-high power and efficiency space traveling-wave tube amplifier power combiner with reduced size and mass for NASA missions," IEEE Trans. Microw. Theory Techn., vol. 57, no. 3, pp. 582-588, Mar. 2009.

[2] F. F. He, K. Wu, W. Hong, L. Han, and X.-P. Chen, "Low-cost 60-GHz smart antenna receiver subsystem based on substrate integrated waveguide technology," IEEE Trans. Microw. Theory Techn., vol. 60, no. 4, pp. 1156-1165, Apr. 2012.

[3] J. Uher, J. Bornemann, and U. Rosenberg, Waveguide Components for Antenna Feed Systems: Theory and CAD. Boston, MA, USA: Artech House, 1993.

[4] H. J. Riblet, "The short-slot hybrid," Proc. IRE, vol. 40, no. 2, pp. 180-184, Feb. 1952.

[5] U. Rosenberg and K. Beis, "Improved narrow-wall short slot coupler design exhibiting significant increased bandwidth and low cost production," in Proc. 31st Eur. Microw. Conf., London, U.K., Sep. 2001, pp. $1-4$.

[6] K. Song, X. Ren, F. Chen, and Y. Fan, "Compact in-phase power divider integrated filtering response using spiral resonator," IET Microw. Antennas Propag., vol. 8, no. 4, pp. 228-234, 2014.

[7] K. Song, F. Chen, F. Zhang, and Y. Fan, "Synthesis and design method of bandpass-response power divider," Microelectron. J., vol. 45, no. 1, pp. 71-77, Jan. 2014.

[8] R. Gomez-Garcia, M. Sanchez-Renedo, and J.-M. Munoz-Ferreras, "Microwave filtering power-distribution networks," in IEEE MTT-S Int. Microw. Symp. Dig., Baltimore, MD, USA, Jun. 2011, pp. 1-4.

[9] F. Lin, Q.-X. Chu, and S. W. Wong, "Design of dual-band filtering quadrature coupler using $\lambda / 2$ and $\lambda / 4$ resonators," IEEE Microw. Wireless Compon. Lett., vol. 22, no. 11, pp. 565-567, Nov. 2012.

[10] C.-H. Wu and C. H. Chen, "Compact LTCC bandpass $180^{\circ}$ hybrid using lumped single-to-differential and single-to-common bandpass filters," in IEEE MTT-S Int. Microw. Symp. Dig., Boston, MA, USA, Jun. 2009, pp. 1473-1476.

[11] W.-M. Chau, K.-W. Hsu, and W.-H. Tu, "Filter-based Wilkinson power divider," IEEE Microw. Wireless Compon. Lett., vol. 24, no. 4, pp. 239-241, Apr. 2014.

[12] H. Uchida, N. Yoneda, Y. Konishi, and S. Makino, "Bandpass directional couplers with electromagnetically-coupled resonators," in IEEE MTT-S Int. Microw. Symp. Dig., San Francisco, CA, USA, May 2006, pp. $1563-1566$.
[13] Y. J. Cheng and Y. Fan, "Compact substrate-integrated waveguide bandpass rat-race coupler and its microwave applications," IET Microw. Antennas Propag., vol. 6, no. 9, pp. 1000-1006, Sep. 2012.

[14] U. Rosenberg and S. Amari, "New power distribution (combination) method with frequency selective properties," in Eur. Microw. Conf., Amsterdam, The Netherlands, Oct./Nov. 2012, Workshop Notes W14: advances of $N$-port networks for space application.

[15] U. Rosenberg, M. Salehi, J. Bornemann, and E. Mehrshahi, "A novel frequency-selective power combiner/divider in single-layer substrate integrated waveguide technology," IEEE Microw. Wireless Compon. Lett., vol. 23, no. 8, pp. 406-408, Aug. 2013.

[16] R. J. Cameron, C. M. Kudsia, and R. R. Mansour, Microwave Filters for Communication Systems: Fundamentals, Design and Applications. New York, NY, USA: Wiley, 2007.

[17] D. M. Pozar, Microwave Engineering, 3rd ed. New York, NY, USA: Wiley, 2005.

[18] S. Amari and U. Rosenberg, "New building blocks for modular design of elliptic and self-equalized filters," IEEE Trans. Microw. Theory Techn., vol. 52, no. 2, pp. 721-736, Feb. 2004.

[19] S. Amari and U. Rosenberg, "The doublet: A new building block for modular design of elliptic filters," in Proc. 32nd Eur. Microw. Conf., Milan, Italy, Oct. 2002, pp. 1-3.

[20] S. Amari and U. Rosenberg, "Synthesis and design of novel in-line filters with one or two real transmission zeros," IEEE Trans. Microw. Theory Techn., vol. 52, no. 5, pp. 1464-1478, May 2004.

[21] U. Rosenberg, "New 'planar' waveguide cavity elliptic function filters," in Proc. 25th Eur. Microw. Conf., Bologna, Italy, Sep. 1995, pp. 524-527.

[22] M. Salehi and E. Mehrshahi, "A closed-form formula for dispersion characteristics of fundamental SIW mode," IEEE Microw. Wireless Compon. Lett., vol. 21, no. 1, pp. 4-6, Jan. 2011.

[23] D. Deslandes, "Design equations for tapered microstrip-to-substrate integrated waveguide transitions," in IEEE MTT-S Int. Microw. Symp. Dig., Anaheim, CA, USA, May 2010, pp. 704-704.

[24] M. Salehi, J. Bornemann, and E. Mehrshahi, "Compact folded substrate integrated waveguide filter with non-resonating nodes for highselectivity bandpass applications," in Proc. 43rd Eur. Microw. Conf., Nuremberg, Germany, Oct. 2013, pp. 155-158.

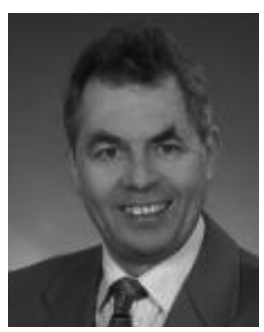

Uwe Rosenberg (M'89-SM'93) received the Dipl. Ing. degree (first-class honors) in electrical engineering (telecommunication technique) from the Fachhochschule der Deutschen Bundespost, Dieburg, Germany, in 1982.

From 1982 to 1983, he was with Hydro Therm, Dieburg, Germany, where he was involved with the design and development of automatic safety and heating control circuits. From 1983 to 1985 , he was with the Technische Hochschule Darmstadt, Darmstadt, Germany, where he was involved with the design and development of experimental installations and software components for microcomputer control systems. In 1985, he joined the Space Division, ANT Nachrichtentechnik GmbH (now Tesat-Spacecom GmbH \& Co. KG), Backnang, Germany, where he was engaged in research and development of microwave filters, multiplexers, and passive subsystems for communications satellites. From 1989 to 2008 he was Head of the Research and Development Laboratory for Passive Microwave Components and Subsystems, Ericsson $\mathrm{GmbH}$ (which was, until December 2005, Marconi Communications GmbH, and formerly Bosch Telecom GmbH, Public Networks Division), Backnang, Germany. During this period, he was in charge of research and development of integrated waveguide transceiver circuitry's, channel branching networks (multiplexers), antenna feed and waveguide (feeder) systems for trunk and access radio applications, mobile base stations, large earth stations, and defense and communications satellites. From 2006 to 2008, he was additionally appointed to Manager of the Antenna Development Team. In 2002, he started his own project and consultancy work for international companies with the design of novel passive microwave designs and subsystems for a variety of applications (earth stations, satellites, millimeter-wave communications equipment, mobile, defense, etc.) providing design, measurement, production, and technology support and advice. Since 2011, he has been the Managing Director with Mician Global Engineering GbR, Bremen, Germany, which he cofounded the same year together with partners. He coauthored Waveguide Components for Antenna Feed Systems: Theory and CAD (Artech House, 1993). He has authored or coauthored over 100 technical papers and has originated more than 50 granted microwave design patents.

Mr. Rosenberg is a member of Verband der Elektrotechnik Elektronik Informationstechnik (VDE), Informationstechnische Gesellschaft (ITG), and Verein 
Deutscher Ingenieure (VDI). He is also a member of the European Microwave Association (EuMA). He is a senior member of the IEEE Microwave Theory and Techniques Society (IEEE MTT-S) and the IEEE Antennas and Propagation Society (IEEE AP-S).

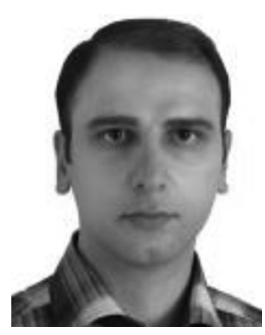

Mehdi Salehi was born in Isfahan, Iran, in 1979. He received the B.Sc. and M.Sc. degrees in electrical engineering from the Isfahan University of Technology (IUT), Isfahan, Iran, in 2001 and 2006, respectively, and the $\mathrm{Ph} . \mathrm{D}$. degree in electrical engineering from Shahid Beheshti University (SBU), Tehran, Iran, in 2013

From 2011 to 2013, he was a Research Assistant with the CADMIC Group, University of Victoria, Victoria, BC, Canada, where he was involved with design, analysis, and synthesis of substrate integrated waveguide (SIW) structures. He is currently a Postdoctoral Fellow with the Coding Signal Transmission (CST) Laboratory, University of Waterloo, Waterloo, ON, Canada, where he is engaged in simulation and hardware implementation of wireless communication systems. His research interests include computer-aided design of RF and microwave structures, numerical methods in electromagnetics, and advanced microwave and millimeter-wave circuits and components.

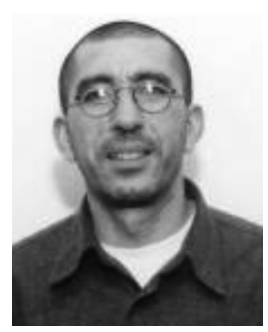

Smain Amari received the D.E.S. degree in physics and electronics from Constantine University, Constantine, Algeria, in 1985, and the Masters degree in electrical engineering and $\mathrm{Ph} . \mathrm{D}$. degree in physics from Washington University, St. Louis, MO, USA, in 1989 and 1994, respectively.

From 1994 to 2000, he was with the Department of Electrical and Computer Engineering, University of Victoria, Victoria, BC, Canada. From 1997 to 1999, he was a Visiting Scientist with the Swiss Federal Institute of Technology, Zurich, Switzerland, and a Visiting Professor in Summer 2001. In 2006, he was a Visiting Professor with the University of Ulm, Ulm, Germany. Since November 2000, he has been with the Department of Electrical and Computer Engineering, Royal Military Col- lege of Canada, Kingston, ON, Canada, where he is currently a Professor. He is interested in numerical analysis, numerical techniques in electromagnetics, applied physics, applied mathematics, wireless and optical communications, computer-aided design (CAD) of microwave components, and the application of quantum field theory in quantum many-particle systems.

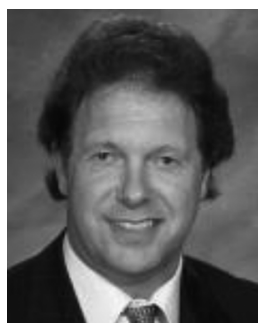

Jens Bornemann (M'87-SM'90-F'02) received the Dipl.-Ing. and Dr.-Ing. degrees in electrical engineering from the University of Bremen, Bremen, Germany, in 1980 and 1984, respectively.

From 1984 to 1985, he was a Consulting Engineer. In 1985, he joined the University of Bremen, as an Assistant Professor. Since April 1988, he has been with the Department of Electrical and Computer Engineering, University of Victoria, Victoria, BC, Canada, where he became a Professor in 1992. From 1992 to 1995 , he was a Fellow of the British Columbia Advanced Systems Institute. In 1996, he was a Visiting Scientis with Spar Aerospace Limited (now MDA Space), Ste-Anne-de-Bellevue, QC, Canada, and a Visiting Professor with the Microwave Department, University of Ulm, Ulm, Germany. From 1997 to 2002, he was a Co-Director of the Center for Advanced Materials and Related Technology (CAMTEC), University of Victoria. In 2003, he was a Visiting Professor with the Laboratory for Electromagnetic Fields and Microwave Electronics, ETH Zurich, Switzerland. He coauthored Waveguide Components for Antenna Feed Systems-Theory and Design (Artech House, 1993). He has authored or coauthored over 300 technical papers. His research activities include RF/wireless/microwave/millimeter-wave components and systems design and field-theory-based modeling of integrated circuits, feed networks, and antennas.

Dr. Bornemann is a Registered Professional Engineer in the Province of British Columbia, Canada. He is a Fellow of the Canadian Academy of Engineering (CAE). He serves on the Editorial Advisory Board of the International Journal of Numerical Modelling. From 1999 to 2002, he was an associate editor for the IEEE TRANSACTIONS ON MicrowaVe THEORY AND TECHNIQUES in the area of microwave modeling and computer-aided design (CAD). From 2006 to 2008, he was an associate editor for the International Journal of Electronics and Communications. From 1999 to 2009, he served on the Technical Program Committee of the IEEE Microwave Theory and Techniques Society (IEEE MTT-S) International Microwave Symposium. 